\title{
Thrombomodulin Alfa
}

National Cancer Institute

\section{Source}

National Cancer Institute. Thrombomodulin Alfa. NCI Thesaurus. Code C128894.

A recombinant, soluble form of the human protein thrombomodulin, with potential anticoagulant activity. Upon administration, thrombomodulin binds to thrombin, which stimulates the activation of protein C. Activated protein C (APC) degrades factor Va and Factor VIIa in the presence of the cofactor protein S, inhibits thrombin formation, and prevents both thrombin-mediated coagulation and further clot formation. 